\title{
Estudo da curva de crescimento de ovinos Santa Inês
}

\section{José Lindenberg Rocha Sarmento ${ }^{1,6}$, Adair José Regazzi ${ }^{2,6}$, Wandrick Hauss de Sousa ${ }^{3}$, Robledo de Almeida Torres ${ }^{4,6}$, Fernanda Cristina Breda', Gilberto Romeiro de Oliveira Menezes ${ }^{5,6}$}

\author{
1 Doutorando em Genética e Melhoramento/UFV, Viçosa-MG, CEP:36571-000. \\ 2 Professor do Departamento de Informática/UFV, Viçosa-MG, CEP:36571-000. \\ 3 Pesquisador III da EMEPA-PB. Caixa postal 275, João Pessoa-PB, CEP:58013-290. \\ ${ }^{4}$ Professor do Departamento de Zootecnia/UFV, Viçosa-MG, CEP:36571-000. \\ ${ }^{5}$ Graduando em Zootecnia/UFV. \\ ${ }^{6}$ Bolsista do CNPq.
}

RESUMO - Utilizaram-se 7.271 registros de pesos do nascimento aos 196 dias de idade de 952 crias de ovinos Santa Inês, controlados de 1983 a 2000, com os objetivos de verificar, entre os modelos Brody, Von Bertalanffy, Logístico, Gompertz e Richards, aquele que melhor descreve a curva média de crescimento e avaliar a influência de efeitos de ambiente sobre os parâmetros estimados. Os parâmetros foram estimados usando-se o procedimento NLIN do SAS. Para verificar se uma única curva seria adequada para descrever o crescimento de machos e fêmeas, nascidos de partos simples ou duplo, aplicou-se um teste de igualdade de parâmetros de modelos não-lineares. Os cinco modelos ajustaram bem os dados de crescimento, entretanto, maior variação dos resíduos foi verificada para os modelos Brody e Logístico. Com base nos desvios médios absolutos dos resíduos, constatou-se que o modelo Gompertz apresentou ajuste médio ligeiramente superior aos modelos Von Bertalanffy e Richards. Machos e fêmeas, nascidos de partos simples e duplo, apresentaram padrões de crescimento divergentes. Os efeitos de grupos contemporâneos, tipo de nascimento e sexo foram importantes fontes de variação para os parâmetros A e K. A correlação estimada entre os parâmetros A e K foi negativa. Melhorias no manejo alimentar devem ser incrementadas, principalmente quanto ao armazenamento de forragem, visando diminuir os efeitos indiretos do clima, o que poderá refletir em menor variação na forma da curva de crescimento.

Palavras-chave: efeitos de ambiente, igualdade de parâmetros, modelo não-linear, ovinos deslanados

\section{Analysis of the growth curve of Santa Ines sheep}

\begin{abstract}
Records of 7,271 weights of 952 lambs measured from birth up to 196 days of age between 1983 and 2000, available from the Agricultural Research Corporation of Paraiba database, were used to model the average growth curve of Santa Ines sheep. The influence of environmental effects on the estimated parameters of the Brody, Von Bertalanffy, Logistic, Gompertz and Richards functions was evaluated using the NLIN procedure of SAS. The growth curve was well fitted by all models but larger residual variation was obtained by the Brody and Logistic models. According to the absolute average residual error, the Gompertz model showed a better fit than the models Von Bertalanffy and Richards. Growth curves differed for males and females, born from single and twin lambing. Contemporary group, type of birth and sex significantly affected the estimation of parameters $\mathrm{A}$ and $\mathrm{K}$. The correlation between parameters $\mathrm{A}$ and $\mathrm{K}$ was negative. Improvements in feeding management and forage storage may decrease the indirect effects of climate and contribute to reduce variation in the growth curves.
\end{abstract}

Key Words: environment effects, equality of parameters, hair sheep, nonlinear model

\section{Introdução}

Em um sistema de produção de ovinos de corte, as características relacionadas ao crescimento são medidas repetidamente em intervalos pré-definidos, caracterizando as chamadas medidas repetidas no tempo. As análises desse tipo de informação podem ser conduzidas de várias formas. Uma possibilidade é a utilização de regressão sobre o tempo, utilizando-se os modelos não-lineares, sendo uma das principais vantagens desse método o agrupamento de várias informações de pesagens associadas à idade, durante o crescimento, em poucos parâmetros biologicamente interpretáveis.

Os modelos não-lineares podem ser utilizados para descrever o crescimento do animal ao longo do tempo, possibilitando avaliar os fatores genéticos e de ambiente que influenciam a forma da curva de crescimento e, desse modo, alterá-la por meio de seleção, ou seja, identificando 
animais com maior velocidade de crescimento, sem alterar o peso adulto, em vez de selecionar animais cada vez maiores.

Vários pesquisadores têm utilizado modelos não-lineares em estudos de crescimento de bovinos de corte (Nobre et al., 1987; Perotto etal., 1992; Oliveira et al., 2000), sobretudo os modelos Brody, Von Bertalanffy, Logístico, Gompertz e Richards.

Questiona-se, no entanto, sobre qual desses modelos é o mais apropriado para descrever o crescimento corporal de ovinos deslanados. McManus et al. (2003), estudando curvas de crescimento de ovinos Bergamácia criados no Distrito Federal, recomendaram que o modelo Logístico deve ser preferido aos de Richards e Brody para ajustar a curva de crescimento desses animais. Santos et al. (2003), por sua vez, compararam os modelos descritos anteriormente no estudo da curva de crescimento de ovinos Santa Inês e verificaram que o de Gompertz proporcionou resultados mais adequados.

Identificado o modelo que melhor se ajustou ao padrão de crescimento, os parâmetros que o descrevem devem ser estudados, identificando-se os fatores que podem influenciá-los, como raça, sexo, tipo de nascimento, época e ano de nascimento e idade da mãe ao parto, possibilitando ajustes no padrão de crescimento. Silva \& Araújo (2000) e Fernandes et al. (2001) verificaram que os efeitos de sexo, tipo de parto, ano de nascimento e idade da mãe ao parto são importantes fontes de variação no crescimento de ovinos deslanados. Espera-se, portanto, que os parâmetros da curva de crescimento sejam afetados por esses efeitos.

Estudos do crescimento de ovinos de corte deslanados, utilizando-se modelos não-lineares, são pouco realizados no Brasil. Objetivou-se com este trabalho verificar, entre os modelos Brody, Von Bertalanffy, Logístico, Gompertz e Richards, aquele que melhor se ajusta aos dados de crescimento. Avaliou-se ainda a influência de fatores de ambiente sobre os parâmetros estimados para descrever a curva de crescimento de ovinos Santa Inês.

\section{Material e Métodos}

Foram utilizados 7.271 registros de pesos ao nascimento (PN), aos 28 (P28), 56(P56), 84(P84), 112 (P112), 140 (P140), 168 (P168) e aos 196 dias de idade (P196) de 952 cordeiros Santa Inês provenientes de um rebanho experimental da Empresa Estadual de Pesquisa Agropecuária do estado da Paraíba (EMEPA-PB), controlados de 1983 a 2000.

A fazenda experimental está localizada no Semi-árido paraibano, caracterizado por duas épocas distintas: uma chuvosa (março a julho) e outra seca (agosto a fevereiro). No período chuvoso, quando, normalmente há maior disponibilidade de alimentos, os animais permaneceram em campos de pastagens nativa e nativa melhorada com capimbuffel (Cenchrus ciliaris) e capim-pangola (Digitaria decumbens). No período de escassez, receberam no cocho suplementação à base de silagem, palma forrageira (Opuntia ficus sp), capim-elefante (Pennisetum purpureum), ração concentrada e sal mineral durante todo o ano. O controle sanitário foi do tipo sistemático. As fêmeas foram cobertas em estação de monta, de modo que o terço final da gestação e parte da lactação coincidissem com o período das chuvas.

Foram utilizados cinco modelos não-lineares para verificar o que melhor descreveu a curva de crescimento média do rebanho, como a seguir: Von Bertalanffy, $Y=A\left(1-B e^{-K t}\right)^{3}+\varepsilon ;$ Brody, $Y=A\left(1-B e^{-K t}\right)+\varepsilon ;$ Gompertz, $Y=A e^{-B e^{-K t}}+\varepsilon ;$ Logístico, $Y=A\left(1+B e^{-K t}\right)^{-1}+\varepsilon ;$ e Richards, $Y=A\left(1-B e^{-K t}\right)^{M}+\varepsilon$, em que $\mathrm{Y}$ é o peso corporal à idade $\mathrm{t}$; $\mathrm{A}$, o peso assintótico quando t tende a mais infinito, ou seja, este parâmetro é interpretado como peso à idade adulta; B, uma constante de integração, relacionada aos pesos iniciais do animal e sem interpretação biológica bem definida. $\mathrm{O}$ valor de $\mathrm{B}$ é estabelecido pelos valores iniciais de $\mathrm{Y}$ e t; $\mathrm{K}$ é interpretado como taxa de maturação, que deve ser entendida como a mudança de peso em relação ao peso à maturidade, ou seja, como indicador da velocidade com que o animal se aproxima do seu tamanho adulto; e M é o parâmetro que dá forma à curva. Sua fixação determina a forma da curva e, conseqüentemente, o ponto de inflexão. Assim, assumindo o ponto de inflexão do modelo de Richards igual a 0 , obtém-se o modelo de Brody; quando igual a 2/3, o modelo Von Bertalanffy; tendendo a 1, o modelo de Gompertz; e se igual a dois, o modelo Logístico. Percebe-se, então, que os primeiros modelos são casos especiais do modelo Richards, que possui quatro parâmetros.

Os parâmetros dos modelos foram estimados pelo método de Gauss Newton modificado por meio do procedimento NLIN do SAS (1999). No caso dos modelos não-lineares, não é possível resolver o sistema de equações formado diretamente, como ocorre no caso linear, uma vez que a resolução do sistema é dependente dos próprios parâmetros a serem estimados. Desta forma, usa-se um processo iterativo para obtenção das estimativas dos parâmetros, o qual começa com valores iniciais, atribuídos aos próprios parâmetros a serem estimados, calcula-se a soma de quadrado do erro e, a cada passo, obtém-se um conjunto de estimativas atualizadas até o procedimento convergir para um vetor final de estimativas, obtendo-se a soma mínima de quadrados do erro. 
Os critérios utilizados para selecionar o modelo que melhor descreveu a curva de crescimento foram: quadrado médio do resíduo (QMR) - calculado dividindo-se a soma de quadrados do resíduo, obtida pelo SAS, pelo número de observações, que é o estimador de máxima verossimilhança da variância residual, para que se pudesse comparar o QMR dos diferentes modelos, visto possuírem número diferentes de parâmetros a serem estimados; coeficiente de determinação $\left(\mathrm{R}^{2}\right)$ - calculado como o quadrado da correlação entre os pesos observados e estimados, que é equivalente a $1-\left(\frac{S Q R}{S Q T_{C}}\right)$ (Souza, 1998), em que SQR é soma de quadrados do resíduo e $\mathrm{SQT}_{\mathrm{C}}$, a soma de quadrados total corrigida pela média; análise gráfica dos resíduos; e desvio médio absoluto dos resíduos (DMA) - estatística proposta neste trabalho para avaliar a qualidade de ajuste, calculado como a seguir:

$$
\text { DMA }=\frac{\sum_{\mathrm{i}=1}^{\mathrm{n}}\left|\mathrm{Y}_{\mathrm{i}}-\hat{\mathrm{Y}}_{\mathrm{i}}\right|}{\mathrm{n}}
$$

em que $Y_{i}$ é o valor observado, $\hat{Y}_{i}$, o valor estimado e $n$ o tamanho da amostra. Quanto menor o valor do DMA, melhor o ajuste.

Após selecionado o modelo, calculou-se a taxa de crescimento absoluta (TCA), obtida a partir da primeira derivada do modelo ajustado, em relação ao tempo $(\partial Y / \partial t)$. A TCA é, na realidade, o ganho de peso obtido por unidade de tempo; como neste caso o tempo está em dia, representa o ganho de peso médio diário, estimado ao longo da trajetória de crescimento, ou seja, a taxa média de crescimento dos animais dentro da população.

Aplicou-se também o teste da razão de verossimilhança para igualdade de parâmetros de modelos não-lineares, segundo Regazzi (2003), para determinar se uma única curva seria adequada para descrever o crescimento de machos e fêmeas (MF), machos nascidos de partos simples ou duplo (MTn1Tn2), fêmeas nascidas de parto simples ou duplo (FTn1Tn2), fêmeas nascidas de parto simples e machos nascidos de parto duplo (FTn1MTn2) e machos nascidos de partos simples e fêmeas de parto duplo (MTn1FTn2).

Em uma etapa seguinte, os parâmetros A, B, K e M estimados para cada animal, dependendo do modelo ajustado, foram analisados utilizando-se os procedimentos GLMe CORR do SAS (1999) para determinação da influência de efeitos de ambiente e das correlações entre os parâmetros estimados.

O modelo estatístico adotado para verificar os fatores de ambiente que poderiam influenciar a forma da curva de crescimento foi:

$$
\mathrm{y}_{\mathrm{ijklm}}=\mu+\mathrm{R}_{\mathrm{i}}+\mathrm{GC}_{\mathrm{j}}+\mathrm{S}_{\mathrm{k}}+\mathrm{TN}_{1}+\mathrm{IDM}_{\mathrm{m}}+\varepsilon_{\mathrm{ijklm}}
$$

em que $\mathrm{y}_{\mathrm{ijk} k \mathrm{~m}}=$ estimativa do parâmetro; $\mu=$ média geral; $\mathrm{R}_{\mathrm{i}}=\mathrm{o}$ efeito aleatório do reprodutor $\mathrm{i} ; \mathrm{GC}_{\mathrm{j}}=$ efeito fixo do grupo contemporâneo $\mathrm{j} ; \mathrm{S}_{\mathrm{k}}=$ efeito fixo do sexo $\mathrm{k} ; \mathrm{TN}_{1}=$ efeito fixo do tipo de nascimento 1 , (nascido de parto simples ou duplo); $\mathrm{IDM}_{\mathrm{m}}=$ efeito fixo da idade da mãe ao parto m; e $\varepsilon_{\mathrm{ijklm}}=$ erro aleatório associado a cada observação.

Os grupos de contemporâneos foram formados pelo ano $(1983, \ldots, 2000)$ e pela estação de nascimento (1 e 2), em que a estação 1 correspondeu ao período chuvoso (março a julho) e a estação 2, ao período seco (agosto a fevereiro). A idade da ovelha ao parto foi agrupada em sete classes: classe 1, Idade $\leq 2 ;$ classe 2, $2<$ Idade $\leq 3$; classe $3,3<$ Idade $\leq 4$; classe $4,4<$ Idade $\leq 5$; classe $5,5<$ Idade $\leq 6$; classe 6 , $6<$ Idade $\leq 7$; e classe 7, Idade $>7$ anos de idade.

\section{Resultados e Discussão}

As estimativas dos parâmetros considerando-se todos os dados, para cada modelo, e os critérios utilizados para avaliar o modelo que melhor descreveu a curva média de crescimento de ovinos Santa Inês são apresentados na Tabela 1.

Com base no $\mathrm{R}^{2}$ (Tabela 1), observa-se que todos os modelos se ajustaram de forma semelhante à curva média de crescimento, como representado na Figura 1, na qual constam as curvas correspondentes às equações ajustadas dos referidos modelos e a curva média observada. Entretanto, ao analisar o QMR, pode-se constatar, apesar da pequena diferença, maior variação para os modelos Brody e Logístico (Figura 1), o que pode ser confirmado pelo gráfico de dispersão dos resíduos ordinários (Figura 2) e pelo DMA (Tabela 1). A distribuição dos resíduos (Figura 2) mostra que o desvio foi maior no ajuste obtido pelos modelos Brody e Logístico e menor no ajuste dos modelos Von Bertalanffy, Gompertz e Richards e que o ajuste desses três últimos foi semelhante, alternando-se ao longo da curva de crescimento quanto ao melhor ajuste.

As diferenças entre os modelos Von Bertalanffy, Gompertz e Richards foram pequenas, permitindo afirmar que se ajustaram de forma satisfatória aos dados de crescimentos de ovinos Santa Inês. Com base no DMA, pequenos desvios entre os modelos foram observados, com menor valor para o modelo Richards, indicando melhor ajuste médio, em comparação aos modelos de Gompertz e Von Bertalanffy. Apesar do bom ajuste, o modelo Richards apresentou problemas quanto à convergência no processo iterativo, ou seja, em aproximadamente $50 \%$ dos animais, a convergência não foi atingida, o que não foi constatado nos 
modelos Gompertz e Von Bertalanffy. Conseqüentemente, o modelo Richards não deve ser escolhido para representar a curva média, visto que a diferença em qualidade de ajuste não supera a dificuldade em atingir convergência, possivelmente por esse modelo necessitar estimar um parâmetro a mais. Dificuldade de convergência com o modelo Richards foi relatada por Braccini Neto et al. (1996), evidenciando que, apesar da maior flexibilidade, por não fixar o ponto de inflexão, esse modelo apresenta maiores dificuldades no processo iterativo em seu ajustamento.
Mesmo com diferenças mínimas de ajuste entre os modelos Gompertz e Von Bertalanffy, o modelo Gompertz obteve DMA ligeiramente inferior e, por isso, foi o escolhido para ajustar a curva média de crescimento. Na literatura consultada, observou-se variação quanto aos modelos indicados para ajustar a curva de crescimento. Bathaei \& Leroy (1996), estudando o crescimento de ovinos no Irã, preferiram o modelo Brody pela simplicidade de interpretação e pela facilidade na estimação de seus parâmetros. Lewis et al. (2002) optaram pelo modelo Gompertz para o

Tabela 1 - Estimativas dos parâmetros ( $\hat{A}, \hat{B}, \hat{K}$ e $\hat{M})$, quadrado médio do resíduo (QMR), coeficiente de determinação $\left(R^{2}\right)$ e desvio médio absoluto (DMA), de acordo com os modelos estudados

Table 1 - Estimate of the parameters ( $\hat{\mathrm{A}}, \hat{\mathrm{B}}, \hat{\mathrm{K}}$ and $\hat{\mathrm{M}}$ ), mean squares of error (MSE), $R$-square $\left(R^{2}\right)$ and absolute average error (AAE) according to the studied models

\begin{tabular}{|c|c|c|c|c|c|c|c|}
\hline \multirow[t]{2}{*}{$\begin{array}{l}\text { Modelo } \\
\text { Model }\end{array}$} & \multicolumn{4}{|c|}{$\begin{array}{c}\text { Estimativa dos parâmetros } \\
\text { Estimate of the parameter }\end{array}$} & \multirow[t]{2}{*}{$\begin{array}{l}\text { QMR } \\
M S E\end{array}$} & \multirow[t]{2}{*}{$\mathrm{R}^{2}$} & \multirow[t]{2}{*}{$\begin{array}{l}\text { DMA } \\
A A E\end{array}$} \\
\hline & $\hat{\mathrm{A}}$ & $\hat{B}$ & $\hat{\mathrm{K}}$ & $\hat{M}$ & & & \\
\hline Bertalanffy & 24,8055 & 0,4799 & 0,0159 & - & 17,2646 & 0,7202 & 0,1569 \\
\hline Brody & 27,4134 & 0,8865 & 0,0094 & - & 17,3723 & 0,7185 & 0,3340 \\
\hline Gompertz & 24,1653 & 1,8731 & 0,0191 & - & 17,2688 & 0,7205 & 0,1552 \\
\hline Logístico & 23,1683 & 4,3772 & 0,0287 & - & 17,4055 & 0,7180 & 0,2865 \\
\hline Richards & 24,5494 & 0,3401 & 0,0171 & 4,6391 & 17,2625 & 0,7202 & 0,1519 \\
\hline
\end{tabular}
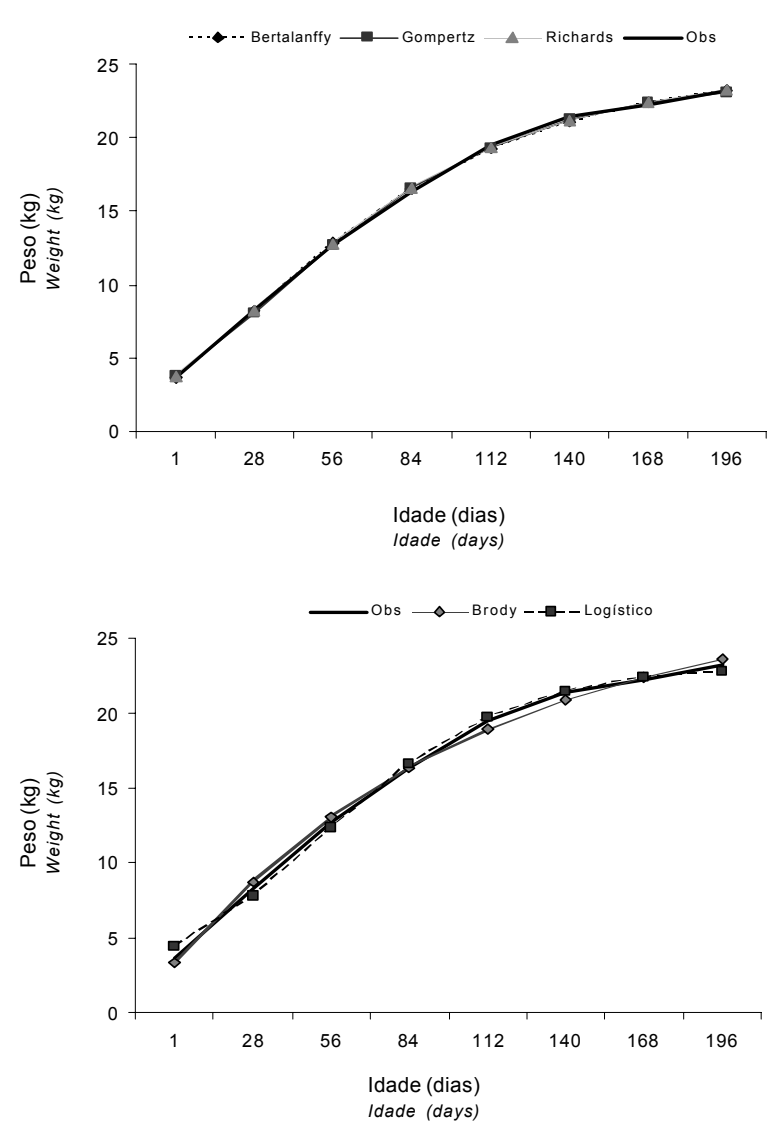

Figura 1 - Curvas de crescimento observada (Obs) e ajustada de acordo com cada modelo.

Figure 1 - Growth curve observed (Obs) and fit according to each model.
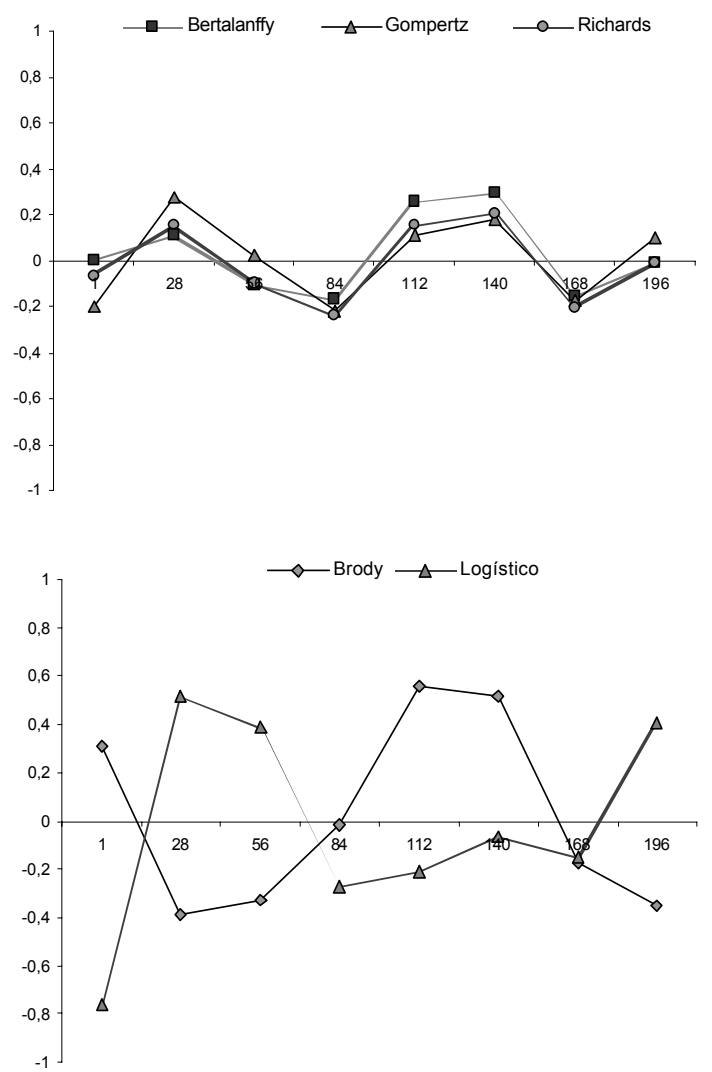

Figura 2 - Dispersão dos resíduos estimados pelos modelos Bertalanffy, Brody, Logístico, Gompertz e Richards.

Figure 2 - Dispersion of error estimateds by models Bertalanffy, Brody, Logistic, Gompertz and Richards. 
estudo da curva de crescimento de ovinos Suffolk sob a alegação de que este modelo apresenta propriedades desejáveis de uma função de crescimento. Entretanto, McManus et al. (2003) compararam os modelos Richards, Brody e Logístico e recomendaram o modelo Logístico para o ajuste da curva de crescimento de ovinos Bergamácia. Essa divergência quanto aos diferentes modelos ajustados é teoricamente compreensível, pois depende do padrão de crescimento dos animais em estudo, ou seja, quando o ponto de inflexão da curva variar de 0 a 1 , o melhor ajuste será obtido com os modelos de Brody e Gompertz; mas quando o ponto de inflexão oscila entre 1 e 2, ficará entre os modelos Gompertz e Logístico (Richards, 1959).

Estes resultados podem ser indicativo, também, de que a avaliação da qualidade de ajuste em modelos não-lineares com base apenas no $\mathrm{R}^{2}$, como corriqueiramente acontece, pode não ser uma boa opção. Ao observar os $\mathrm{R}^{2}$ para os diferentes modelos (Tabela 1), verifica-se que todos foram muito próximos, praticamente não indicando diferença entre os cinco modelos. Assim, pelo $\mathrm{R}^{2}$, qualquer um dos modelos teria o mesmo ajuste. Ao observar o DMA (Tabela 1) e a dispersão dos resíduos (Figura 2), constata-se, no entanto, maior variação no ajuste obtido pelos modelos Brody e Logístico e que o Logístico é ligeiramente melhor que o Brody, fato imperceptível quando se observa o $\mathrm{R}^{2}$.

As taxas de crescimento absoluto (TCA), obtidas pela primeira derivada do modelo Gompertz em relação ao tempo

$$
\left(\frac{\partial Y}{\partial t}=A K B e^{-K t} e^{-B e^{-K t}}\right) \text {, }
$$

são apresentadas na Figura 3. A TCA foi crescente até atingir o máximo, em torno de $0,170 \mathrm{~kg}$ por dia $\left(K A e^{-1}\right)$, e depois decresceu com a idade. O ponto em que a função muda de crescente para decrescente, ou seja, o ponto de

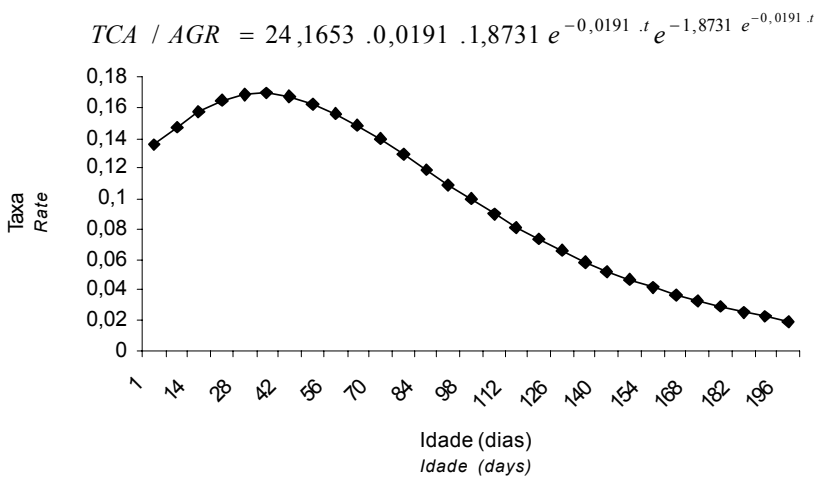

Figura 3 - Comportamento da taxa de crescimento absoluto (TCA) estimada pelo modelo Gompertz.

Figure 3 - Behavior of the absolute growth rate (AGR) estimated by Gompertz model. inflexão da curva, foi atingido aos 52 dias de idade $\left(K^{-1}\right)$ com peso de, aproximadamente, $8,9 \mathrm{~kg}\left(A e^{-1}\right)$. Esses resultados indicam que a taxa máxima de crescimento foi alcançada muito cedo, antes dos dois meses de idade, e que a essa idade o animal já atingiu a velocidade máxima de crescimento. As TCA no final do período estudado foram muito pequenas, comprovando que, em torno dos seis meses de idade, os animais praticamente não ganharam peso.

A TCA máxima a essa idade pode ser indicativo de limitação no crescimento, decorrente da insuficiente disponibilidade de alimentos em idades posteriores, o que, possivelmente, foi causa principal da sazonalidade na produção de forragem. Aos 60 dias de idade, quando a TCA começa a diminuir, o cordeiro ainda está sob cuidados maternos, mas provavelmente o leite produzido pela mãe já não é mais suficiente para atender às exigências de crescimento. Após a desmama (112 dias, aproximadamente), a TCA continuou decrescendo, o que pode ser resultado de manejo inadequado para acompanhar a maior demanda de nutriente à medida que o animal cresceu. Entretanto, esses resultados podem indicar que esses animais se adaptaram ao semi-árido, adquirindo este padrão de crescimento, isto é, velocidade de crescimento maior quando mais jovens e menor em idades mais avançadas, proporcionando menores pesos à maturidade, o que parece ser biologicamente compatível com as condições climáticas a que foram submetidos.

$\mathrm{O}$ teste de igualdade de parâmetros aplicado ao modelo de Gompertz encontra-se na Tabela 2. Comparando-se as curvas de machos e fêmeas (MF), verificou-se que a hipótese $\mathrm{A} 1=\mathrm{A} 2=\mathrm{A}$ foi rejeitada, ou seja, o parâmetro $\mathrm{A}$, relacionado ao peso à maturidade, foi diferente para ambos os sexos, sugerindo que duas curvas são necessárias, uma para machos e outra para fêmeas. Quanto aos parâmetros B e $\mathrm{K}$, as hipóteses não foram rejeitadas, indicando que, possivelmente, possuem a mesma taxa de crescimento (K) e mesma constante de integração (B).

Quanto ao tipo de nascimento, constatou-se que as hipóteses que testaram a igualdade dos parâmetros A e K foram rejeitadas para MTn1 $\mathrm{Tn} 2$, assim como para FTn1Tn2, evidenciando que, mesmo sendo do mesmo sexo (macho ou fêmea), os animais apresentaram padrão de crescimento diferenciado, visto terem nascido de uma matriz gestando uma ou duas crias. Os valores do $\chi^{2}$ calculado (Tabela 2) para os parâmetros $\mathrm{A} \mathrm{e} \mathrm{K}$ foram maiores para as fêmeas que para os machos, demonstrando que o efeito de tipo de nascimento proporcionou maior variação nas fêmeas.

Comparando os parâmetros A e K para as fêmeas nascidas de parto simples e os machos de parto duplo (FTn1MTn2), verificou-se inversão no padrão de crescimento, como 
Tabela 2 - Resumo do teste de igualdade de parâmetros aplicado ao modelo de Gompertz para machos e fêmeas, combinados ao tipo de nascimento

Table 2 - $\quad$ Summary of the test for parameters equality applied to Gompertz model for males and females, agreements to type of birth

\begin{tabular}{|c|c|c|c|c|c|c|c|c|c|c|c|}
\hline \multirow[t]{2}{*}{ Hipóteses $\left(\mathrm{H}_{0}\right)$} & \multirow[t]{2}{*}{ GL } & \multicolumn{2}{|c|}{ MF } & \multicolumn{2}{|c|}{$\operatorname{MTn} 1 \operatorname{Tn} 2$} & \multicolumn{2}{|c|}{ FTn 1 Tn 2} & \multicolumn{2}{|c|}{ FTn $1 M T n 2$} & \multicolumn{2}{|c|}{ MTn1FTn2 } \\
\hline & & $\chi_{\text {calc }}^{2}$ & Val-P & $\chi_{\text {calc }}^{2}$ & Val-P & $\chi_{\text {calc }}^{2}$ & Val-P & $\chi_{\text {calc }}^{2}$ & Val-P & $\chi_{\text {calc }}^{2}$ & Val-P \\
\hline $\mathrm{A}_{1}=\mathrm{A}_{2}=\mathrm{A}$ & 1 & 25,30 & 0,001 & 17,66 & 0,001 & 25,03 & 0,001 & 4,45 & 0,03 & 36,79 & 0,001 \\
\hline $\mathrm{B}_{1}=\mathrm{B}_{2}=\mathrm{B}$ & 1 & 0,65 & 0,42 & 3,092 & 0,07 & 1,87 & 0,17 & 5,12 & 0,02 & 0,75 & 0,17 \\
\hline $\mathrm{K}_{1}=\mathrm{K}_{2}=\mathrm{K}$ & 1 & 0,71 & 0,40 & 12,99 & 0,001 & 20,13 & 0,001 & 11,79 & 0,001 & 20,17 & 0,001 \\
\hline
\end{tabular}

$\chi^{2}$ calc $=$ valor do qui-quadrado calculado; Val-P = valor de probabilidade.

$\chi^{2}{ }_{\text {calc }}=$ value from chi-squared calculated; Val- $P=P$-value .

conseqüência do efeito do tipo de nascimento, ou seja, neste caso específico, as fêmeas apresentaram peso assintótico e taxa de crescimento maiores que os machos. Para os machos nascidos de parto simples e as fêmeas de parto duplo, o resultado obtido foi o esperado, pois, além do dimorfismo sexual, houve contribuição do tipo de nascimento, evidenciando a superioridade dos machos. A partir desses resultados, pode-se constatar a importância do efeito de tipo de nascimento, que pode ser confirmada com os valores do $\chi^{2}$ calculado. Para machos nascidos de parto simples e fêmeas de parto duplo, os valores do $\chi^{2}$ calculados para os parâmetros A e K foram 36,79 e 20,17, respectivamente, ao passo que os calculados para as fêmeas nascidas de parto simples e machos de parto duplo foram, na mesma ordem, 4,45 e 11,79 (significativos, $\mathrm{P}<0,05$ ), indicando que o efeito de tipo de nascimento foi capaz de anular o efeito de dimorfismo sexual e proporcionar maior crescimento para as fêmeas.

A análise de variância revelou efeito aleatório de reprodutor significativo $(\mathrm{P}<0,05)$ para o parâmetro $\mathrm{K}$. Este efeito pode ser atribuído a fatores genéticos, o que só poderia ser constatado com a estimação de componentes de variância e parâmetros genéticos.

O efeito de grupo contemporâneo foi significativo $(\mathrm{P}<0,05)$ para os três parâmetros estimados, o que contribuiu com mais de $40 \%$ da soma de quadrados de todos os efeitos considerados no modelo, evidenciando a influência da estacionalidade na produção de alimentos sobre os parâmetros estimados e, conseqüentemente, sobre o padrão de crescimento dos cordeiros. Esse resultado indica que, mesmo com o manejo adotado na fazenda, os efeitos indiretos do clima não foram anulados, mas possivelmente amenizados.

Os parâmetros $\mathrm{A}$ e $\mathrm{K}$ também foram influenciados $(\mathrm{P}<0,05)$ pelo sexo do cordeiro (Tabela 3 ), semelhantemente ao observado por Bathaei \& Leroy (1996) e McManus et al. (2003), em que os parâmetros foram ajustados pelo modelo Brody. As médias das estimativas dos parâmetros A e K foram maiores para os machos (Tabela 3), o que pode confirmar o dimorfismo sexual para ganho de peso.
O tipo de nascimento afetou significativamente as estimativas dos parâmetros (Tabela 3). As estimativas de A e K para animais nascidos de parto simples foram superiores às obtidas para os nascidos de parto duplo, contudo, McManus et al. (2003) constataram que este efeito não influenciou o peso à maturidade. A contribuição do tipo de nascimento para a variação total foi de aproximadamente $15 \%$ para os parâmetros A e K e próximo de $10 \%$ para o B, mostrando ser um efeito importante e que deve ser considerado no crescimento de ovinos, principalmente se criados ao pé da mãe.

Ressalta-se que a interação sexo x tipo de nascimento foi inicialmente incluída no modelo estatístico, mas a análise de variância revelou que este efeito foi não significativo $(\mathrm{P}>0,05)$, motivo pelo qual foi desconsiderada. Entretanto, pelos resultados obtidos pelo teste de igualdade de parâmetros de modelos de regressão não-linear, constatou-se que esta interação existe e que foi importante, evidenciando que fêmeas nascidas de partos simples apresentaram peso assintótico e taxa de crescimento superiores aos de machos nascidos de parto duplo. Semelhantemente, Bathaei \& Leroy (1996) relataram que as interações não foram importantes, mas, entre os fatores de ambiente considerados, o sexo e o tipo de nascimento foram as principais fontes de variação.

A idade da mãe ao parto influenciou $(\mathrm{P}<0,05)$ os três parâmetros da curva, provavelmente em razão das mudanças na produção de leite e dos cuidados maternos sobre a prole, em função da idade. Bathaei \& Leroy (1996) constataram que a idade da mãe não influenciou os parâmetros A e K. Ovelhas mais novas tenderam a produzir cordeiros com maior peso assintótico (Tabela 3), ao passo que as mais velhas produziram animais com menores pesos à maturidade. Porém, para o parâmetro K, os valores estimados revelaram tendência de comportamento quadrático, ou seja, os cordeiros filhos de ovelhas mais jovens e mais velhas tenderam a apresentar menores taxas de crescimento, enquanto os filhos de ovelhas em idade intermediária (2 a 5 anos) tenderam a taxas de crescimento maiores.

A correlação entre as estimativas dos parâmetros A e $K$ foi negativa (Tabela 4), corroborando aos achados de 
Bathaei \& Leroy (1996) e McManus et al. (2003). Uma interpretação biológica para essa correlação seria que animais com maiores taxas de crescimento têm menor probabilidade de atingir maiores pesos à maturidade que aqueles que crescem mais lentamente no início da vida. Ou seja, animais que atingem a maturidade mais pesados tendem a menor taxa de crescimento. Deve-se ressaltar que esta relação é, biologicamente, a mais importante.

A correlação entre B e K foi negativa e uma possível interpretação biológica para esta correlação seria que cordeiros desmamados mais leves (valores altos de B) tendem a menores taxas de crescimento relativo (quociente entre ganho de peso e peso), e conseqüentemente a menores taxas de

Tabela 3 - Médias de quadrados mínimos para as estimativas dos parâmetros pelo modelo Gompertz de acordo com o sexo (S), tipo de nascimento (TN) e a idade da mãe ao parto (IDM)

Table 3 - Least squares means for estimate of the parameters by Gompertz model according to sex (S), type of birth (TB) and age of dam at lambing (ADL)

\begin{tabular}{|c|c|c|c|}
\hline \multirow[t]{2}{*}{$\begin{array}{l}\text { Fonte de variação } \\
\text { Source of variation }\end{array}$} & \multicolumn{3}{|c|}{$\begin{array}{l}\text { Média das estimativas dos parâmetros } \\
\text { Average of the estimate of the parameters }\end{array}$} \\
\hline & $\hat{\mathrm{A}}$ & $\hat{\mathrm{B}}$ & $\hat{\mathrm{K}}$ \\
\hline \multicolumn{4}{|l|}{ S } \\
\hline Macho (Male) & 25,58 a & 2,01 a & $0,0202^{a}$ \\
\hline Fêmea (Female) & 24,51 b & $1,98^{\mathrm{a}}$ & $0,0193 \mathrm{~b}$ \\
\hline \multicolumn{4}{|l|}{$\mathrm{TN}(T B)$} \\
\hline Simples (Single) & $26,66^{a}$ & $1,93 \mathrm{~b}$ & $0,0214^{a}$ \\
\hline Duplo (Twin) & $23,43 \mathrm{~b}$ & $2,06^{\mathrm{a}}$ & 0,0181 b \\
\hline \multicolumn{4}{|l|}{$\operatorname{IDM}(A D L)$} \\
\hline Idade $\leq 2$ & $25,60^{\mathrm{a}}$ & $2,05^{\mathrm{a}}$ & $0,0197 \mathrm{abc}$ \\
\hline $2<$ idade $\leq 3$ & 25,52 a & $2,00 \mathrm{ab}$ & $0,0206^{a b}$ \\
\hline $3<$ idade $\leq 4$ & $25,34^{\mathrm{a}}$ & 2,01 a & $0,0203 \mathrm{ab}$ \\
\hline $4<$ idade $\leq 5$ & 25,49 a & $2,00 \mathrm{ab}$ & $0,0210^{\mathrm{a}}$ \\
\hline $5<$ idade $\leq 6$ & $24,86^{a b}$ & $1,92 \mathrm{~b}$ & $0,0194 \mathrm{bc}$ \\
\hline $6<$ idade $\leq 7$ & $25,26 \mathrm{ab}$ & $2,02^{a}$ & $0,0187^{\mathrm{c}}$ \\
\hline Idade $>7$ & $23,26 \quad b$ & $1,96 \mathrm{ab}$ & $0,0185^{\mathrm{c}}$ \\
\hline
\end{tabular}

Valores seguidos de pelo menos uma mesma letra dentro de cada fonte de variação, na coluna, não diferiram significativamente $(P>0,05)$ pelo teste t. Values followed by at least a same letter within each variation source, in the column, did not differ significant $(P>0.05)$ by test $t$.

Idade (age). maturidade (menores valores de $\mathrm{K}$ ), que aqueles desmamados mais pesados. A correlação positiva entre A e B pode indicar que cordeiros mais pesados em idades que antecedem o desmame e na ocasião do desmame tendem a atingir maiores pesos à maturidade. Bathaei \& Leroy (1996), estudando ovinos no Irã, verificaram resultados contrários aos deste estudo.

O parâmetro A apresentou correlação positiva e crescente aos pesos do nascimento aos 196 dias de idade (Tabela 4), indicando que, ao se enfatizar pesos, principalmente após o desmame, pode ocorrer aumento indireto no peso à maturidade. Contudo, para o parâmetro K, a correlação foi crescente até o peso aos 84 dias, decrescendo a partir dessa idade e chegando a ser negativa no peso aos 196 dias de idade, confirmando a correlação negativa entre A e K.

Esses resultados podem ser úteis para produtores ou para utilização em programas de melhoramento possíveis de implementação, auxiliando na estruturação do manejo a ser adotado, de modo a permitir que os animais expressem seu padrão de crescimento com a menor interferência possível do ambiente a que estão submetidos. Entretanto, novos estudos devem ser conduzidos visando avaliar como os parâmetros da curva de crescimento podem ser usados em programas de seleção.

\section{Conclusões}

O modelo Gompertz apresentou ajuste médio superior e, portanto, deve ser preferido aos demais modelos estudados para descrição da curva média de crescimento. Machos e fêmeas, nascidos de partos simples ou duplo, apresentaram padrão de crescimento divergente, indicando a necessidade de curvas diferentes para descrever seus crescimentos. Melhorias no manejo alimentar devem ser implementadas, principalmente quanto ao armazenamento de forragem, visando diminuir os efeitos indiretos do clima, o que poderá resultar em menor variação na forma da curva de crescimentos e em melhores taxas de crescimento em idades mais jovens.

Tabela 4 - Correlações entre as estimativas dos parâmetros da curva de crescimento de Gompertz e pesos do nascimento aos 196 dias de idade

Table 4 - $\quad$ Correlations between the estimate of the parameters of the growth curve of Gompertz and weights from birth to 196 days of age

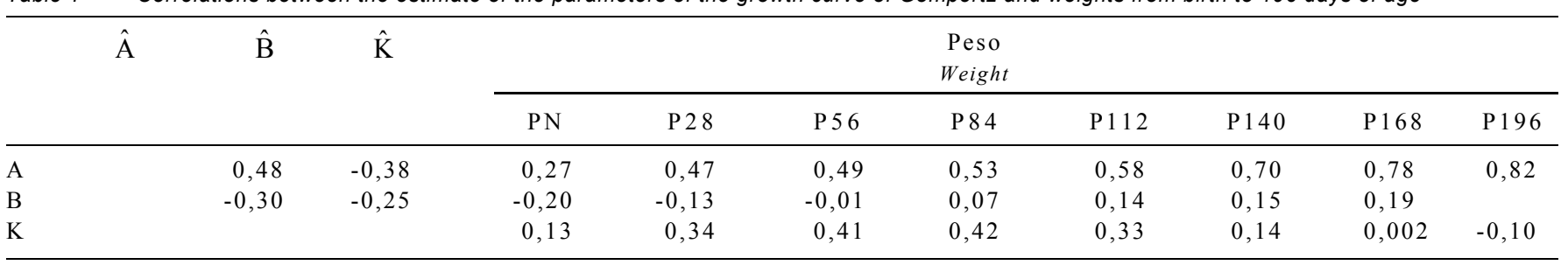




\section{Agradecimento}

À CAPES, pela bolsa de estudos concedida ao primeiro e quarto autores durante parte de seus cursos de doutoramento.

À Empresa Estadual de Pesquisa Agropecuária do Estado da Paraíba (EMEPA-PB), pela cessão dos dados.

\section{Literatura Citada}

BATHAEI, S.S.; LEROY, P.L. Growth and mature weight of Mehraban Iranian fat-taliled sheep. Small Ruminants Research, v.22, p.155-162, 1996.

BRACCINI NETO, J.; DIONELLO, N.J.L.; SILVEIRA JR., P. et al. Análise de curvas de crescimento de aves de postura. Revista Brasileira de Zootecnia, v.25, n.6, p.1062-1073, 1996.

FERNANDES, A.A.O.; BUCHANAN, D.; SELAIVE-VILLARROEL, A.B. Avaliação dos fatores ambientais no desenvolvimento corporal de cordeiros deslanados da raça Morada Nova. Revista Brasileira de Zootecnia, v.30, n.5, p.1460-1465, 2001.

LEWIS, R.M.; EMMANS, G.C.; DINGWALL, W.S. et al. A description of the growth of sheep and its genetic analysis. Animal Science, v.74, p.51-62, 2002.

MCMANUS, C.; EVANGELISTA, C.; FERNANDES, L.A.C. et al. Curvas de crescimento de ovinos Bergamácia criados no Distrito Federal. Revista Brasileira de Zootecnia, v.32, n.5, p.1207$1212,2003$.

NOBRE, P.R.; ROSA, A.N.; SILVA, L.O.C. et al. Curvas de crescimento de gado Nelore ajustadas para diferentes frequências de pesagens. Pesquisa Agropecuária Brasileira, v.22, n.9/ 10, p.1027-1037, 1987.
OLIVEIRA, H.N.; LÔBO, R.B.; PEREIRA, C.S. Comparação de modelos não-lineares para descrever o crescimento de fêmeas da raça Guzerá. Pesquisa Agropecuária Brasileira, v.35, p.1843-1851, 2000 .

PEROTTO, D.; CUE, R.I.; LEE, A.J. Comparison of nonlinear functions for describing the growth curve of three genotypes of dairy cattle. Canadian Journal of Animal Science, v.72, p.753-782, 1992 .

REGAZZI, A.J. Teste para verificar a igualdade de parâmetros e a identidade de modelos de regressão não-linear. Revista Ceres, v.50, n.287, p.9-26, 2003.

RICHARDS, F.J. A flexible growth function for empirical use. Journal of Experimental Botany, v.10, p.290-300, 1959.

SANTOS, C.L.; PÉREZ, J.R.O.; MUNIZ, J.A. et al. Parâmetros da curva de crescimento de ovinos Santa Inês. In: REUNIÃO ANUAL DA SOCIEDADE BRASILEIRA DE ZOOTECNIA, 40., 2003, Santa Maria. Anais... Santa Maria: Sociedade Brasileira de Zootecnia, 2003, CD-ROM.

STATISTICAL ANALYSIS SYSTEM - SAS. User's guide: statistics. Version 8.0. Cary: 1999.

SILVA, F.L.R.; ARAÚJO, A.M. Características de reprodução e de crescimento de ovinos mestiços Santa Inês, no Ceará. Revista Brasileira de Zootecnia, v.29, n.6, p.1712-1720, 2000.

SOUZA, G.S. Introdução aos modelos de regressão linear e não-linear. Brasília: Serviço de Produção de Informação, 1998. $505 \mathrm{p}$.

Recebido: $28 / 10 / 04$ Aprovado:08/11/05 